\title{
Model of a Dipole Antenna in Free-Space and Near a Perfectly Electrical Conducting Plate
}

\author{
Nobby Stevens and Luc Martens, Member, IEEE
}

\begin{abstract}
In this paper, an accurate method is developed to model dipole antennas in free-space or near an infinite perfectly electrical conducting plate. The magnetic field integral equation is implemented and point-matching is used to construct the interaction matrix. The edges of the cylinder, which can cause numerical instabilities in the simulated current distribution, are getting a special treatment by choosing a specific set of basis functions. The excitation is modeled based on the equivalence principle and leads to the application of a magnetic surface current over the gap region. The exact kernel of the integral equation is applied and all possible current components are taken into account. When the antenna is close to a conducting plate, a strong modification of the surface current distribution is observed. It is demonstrated that for these configurations, the incorporation of the circumferential component of the surface current distribution is needed. At larger distances, the circumferential surface current can be neglected in the total distribution. The only approximation (or rather assumption) we made is that we impose a longitudinal directed electrical field over the boundary of the gap region. The results are verified experimentally.
\end{abstract}

Index Terms -Dipole antennas, magnetic field integral equation (MFIE), method of moments (MoM), scattering.

\section{INTRODUCTION}

$\mathbf{T}$ HE study of dipoles is an old topic with still many practical applications. Dipole antennas are used for calibration of other antennas, for electromagnetic compatibility (EMC) testing and are the basic components of wireless base station antennas. Over the recent century, several techniques and methods have been developed to solve the configuration of a finite circular dipole antenna in free-space [1]-[17] or near a conducting half space [18]. Due to the open boundary character, the vast majority of these methods deal with the solution of an integral equation. If certain restrictions with regard to the diameter of the dipole are taken into account, the integral equations of Hallén or Pocklington prove to be simple and efficient to calculate the main far-field properties of the dipole. For small diameters $(<0.01 \lambda)$, the kernel of the correct integral equation can be reduced to a simpler form, which leads to an integral equation that can be solved easily by the method of moments or by iteration. In this approximation, one assumes that the current flows only in the direction of the wire axis. The charge and current contribution on the end caps are ignored and the boundary

Manuscript received March 17, 2003; revised August 10, 2004.

N. Stevens was with the Department of Information Technology, Ghent University, B-9000 Ghent, Belgium. He is now with Agilent EEsof EDA, Ghent, Belgium.

L. Martens is with Interuniversity Microelectronics Centre (IMEC), B-3030 Leuven, Belgium and also with the Department of Information Technology (INTEC), Ghent University, B-9000 Ghent, Belgium (e-mail: luc.martens@intec.UGent.be).

Digital Object Identifier 10.1109/TAP.2005.846724 condition for the electrical field is only applied to the axial component at the wire surface. Previous work [19] shows that if the radius exceeds the critical value of $\lambda / 100$ (rule of thumb), the solution of the problem becomes much more complicated.

A difficulty in the modeling of dipole antennas is the excitation. A popular excitation is the delta-source. A good model for the source is presented by Cui and Chew [20]. It is based on the equivalence principle and it was shown that this type of source, in combination with the reciprocity theorem, leads to a variational formulation of the input impedance. The main restrictions of the model of Cui [20] are the limitation of the diameter and the fact that the caps are not incorporated in the model. The effect of the end caps was studied by Huffman and Werner [21]. The edge singularities were taken into account by using a ring of charge.

In our work, all possible current components are calculated. We have not found any publication where the curved surface of a dipole is followed exactly while all possible surface currents are taken into account. It is demonstrated in this paper that for a configuration of a dipole near a PEC plate, one needs to incorporate all possible surface currents for small separation distances.

We have decided to use the magnetic field integral equation (MFIE) instead of the electric field integral equation (EFIE) for several reasons [22], [23]. The first reason is that the EFIE is a Fredholm integral equation of the first kind, whereas the MFIE is one of the second kind. The MFIE involves the sum of a compact operator and a constant operator, for which it is formally possible to prove convergence [24]. This is not the case for the EFIE. A second reason is related to numerical considerations. The system matrix of the EFIE tends to become less diagonally dominant when the number of mesh points is increased. The self-patch term of the MFIE contributes in the same way to the diagonal elements of the interaction matrix, irrespective of the mesh size. As a consequence, the interaction matrix will become more diagonally dominant as the number of match points increases, which leads to a higher numerical stability and a better condition number compared to the EFIE for the same number of unknowns. A disadvantage of the MFIE is that it can only be used for closed surfaces, whereas the EFIE can also be applied for open bodies. As one or several dimensions of the body become small (relative to the wavelength), it is more difficult to obtain convergence with the MFIE, as stated by Poggio and Miller [25]. Nevertheless, even for thin wires, the MFIE is proven to deliver accurate results as will be demonstrated. The excitation model we use is based on the equivalence principle. In the first part of this paper, a model of a dipole antenna in free-space is described. The current distribution is shown and compared to results from other simulation tools. It is demonstrated that for dipoles in free-space, the circumferential surface currents are 


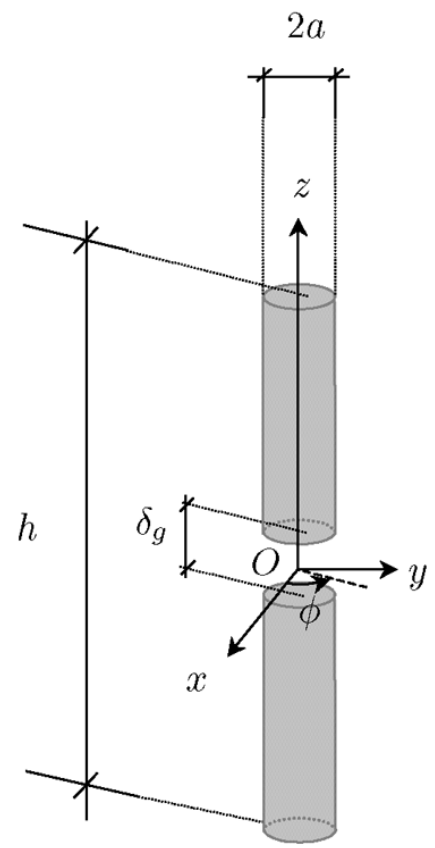

Fig. 1. Configuration of a dipole with the corresponding notations.

equal to zero. In the second part of this paper, we will model the behavior of a dipole when it is not in free-space, but close to another PEC object. As an example, we will consider the configuration of a dipole at various distances from an infinite PEC plane. It is observed that the input admittance is strongly dependent on the distance from this plane. The surface current distribution is not rotational symmetric anymore. The results of the model are confirmed by experimental data.

\section{Dipole IN Free-Space: Model AND Results}

\section{A. Model}

Let us consider Fig. 1, where a dipole in free-space with the corresponding notations is shown. The total height of the dipole with radius $a$ is $h$ while the gap width is $\delta_{g}$. A difficult task in the correct modeling of a cylindrical dipole is the source model. The correct behavior of the field within the gap region is dependent on the practical excitation. Nevertheless, we do not need to know the excitation within the gap region; the equivalence principle allows us to omit the complex behavior. The only component that one has to know is the tangential field component of the electric or magnetic field at the boundary of a closed volume [26], [27]. The closed volume $V^{\prime}$ we consider is of course the gap region. It is defined as (corresponding to the notations of Fig. 1): $|z| \leq\left(\delta_{g} / 2\right), r \leq a$, and for all $\phi$. We suppose that the electric field over surface $S^{\prime}$ of $V^{\prime}$ is known. If a voltage source of $1 \mathrm{~V}$ is applied over the gap region, the applied electric field at the surface is approximated by

$$
\mathbf{E}(\mathbf{r})=-1 / \delta_{g} \mathbf{u}_{z} \cdot
$$

Based on the uniqueness theorem and equivalence principle, the surface $S^{\prime}$ can be replaced by a perfect conductor carrying a magnetic surface current $\mathbf{K}_{s}(\mathbf{r})$ defined by [26], [27]

$$
\mathbf{K}_{s}(\mathbf{r})=\mathbf{E}(\mathbf{r}) \times \mathbf{u}_{\mathbf{n}}(\mathbf{r})=-\frac{\mathbf{u}_{z}}{\delta_{g}} \times \mathbf{u}_{\mathbf{r}}(\mathbf{r})=-\frac{\mathbf{u}_{\phi}(\mathbf{r})}{\delta_{g}}
$$

with $\mathbf{u}_{\mathbf{n}}(\mathbf{r})$ the outward normal of the surface $S^{\prime}$. We remark that the solution is not valid inside $V^{\prime}$. So a dipole in free-space can be modeled as a PEC rod with a magnetic surface current excitation imposed over the gap region. We have not investigated large gaps (relative to the wavelength), but for practical dipoles used at their operating frequency, the gaps are always small. We now formulate the coupled set of Fredholm integral equations of the second kind since they are of importance further in this work. If the classical cylindrical coordinates are introduced in Fig. 1 (with $\phi$ the angle starting at the $x$ axis and increasing counterclockwise), the following set of equations is valid for locations $r$ on the cylindrical surface ( $S_{\mathrm{cs}}$ stands for the cylindrical surface and $S_{c}$ for the surface of the caps)

$$
\begin{aligned}
& \frac{1}{2} J_{z}(\mathbf{r})-f_{S_{\mathrm{cs}}} J_{z}\left(\mathbf{r}^{\prime}\right)\left[G_{\phi^{\prime}}\left(\mathbf{r}, \mathbf{r}^{\prime}\right) \sin \left(\phi-\phi^{\prime}\right)\right. \\
& \left.+G_{r^{\prime}}\left(\mathbf{r}, \mathbf{r}^{\prime}\right) \cos \left(\phi-\phi^{\prime}\right)\right] a d \phi^{\prime} d z^{\prime} \\
& +\int_{S_{\mathrm{cs}}} J_{\phi}\left(\mathbf{r}^{\prime}\right) G_{z^{\prime}}\left(\mathbf{r}, \mathbf{r}^{\prime}\right) \sin \left(\phi-\phi^{\prime}\right) a d \phi^{\prime} d z^{\prime} \\
& +\int_{S_{c}} J_{r}\left(\mathbf{r}^{\prime}\right) G_{z^{\prime}}\left(\mathbf{r}, \mathbf{r}^{\prime}\right) \cos \left(\phi-\phi^{\prime}\right) \\
& +J_{\phi}\left(\mathbf{r}^{\prime}\right) G_{z^{\prime}}\left(\mathbf{r}, \mathbf{r}^{\prime}\right) \sin \left(\phi-\phi^{\prime}\right) r^{\prime} d \phi^{\prime} d r^{\prime} \\
& \quad=\mathbf{u}_{z} \cdot\left[\mathbf{u}_{r}(\mathbf{r}) \times \mathbf{H}_{\mathrm{inc}}(\mathbf{r})\right] \\
& \frac{1}{2} J_{\phi}(\mathbf{r})-f_{S_{\mathrm{cs}}} J_{\phi}\left(\mathbf{r}^{\prime}\right) G_{r^{\prime}}\left(\mathbf{r}, \mathbf{r}^{\prime}\right) a d \phi^{\prime} d z^{\prime} \\
& +\int_{S_{c}} J_{r}\left(\mathbf{r}^{\prime}\right) G_{\phi^{\prime}}\left(\mathbf{r}, \mathbf{r}^{\prime}\right)-J_{\phi}\left(\mathbf{r}^{\prime}\right) G_{r^{\prime}}\left(\mathbf{r}, \mathbf{r}^{\prime}\right) r^{\prime} d \phi^{\prime} d r^{\prime} \\
& \quad=\mathbf{u}_{\phi}(\mathbf{r}) \cdot\left[\mathbf{u}_{r}(\mathbf{r}) \times \mathbf{H}_{\mathrm{inc}}(\mathbf{r})\right] .
\end{aligned}
$$

For the observation point $\mathbf{r}$ located on the cap surfaces, (5) and (6) are applicable. The lower or upper sign is used when $\mathbf{r}$ is respectively on the bottom or top cap

$$
\begin{aligned}
& \frac{1}{2} J_{r}(\mathbf{r}) \pm \int_{S_{\mathrm{cs}}} J_{z}\left(\mathbf{r}^{\prime}\right)\left[G_{r^{\prime}}\left(\mathbf{r}, \mathbf{r}^{\prime}\right) \cos \left(\phi-\phi^{\prime}\right)\right. \\
& \left.+G_{\phi^{\prime}}\left(\mathbf{r}, \mathbf{r}^{\prime}\right) \sin \left(\phi-\phi^{\prime}\right)\right] a d \phi^{\prime} d z^{\prime} \\
& \mp \int_{S_{\mathrm{cs}}} J_{\phi}\left(\mathbf{r}^{\prime}\right) G_{z^{\prime}}\left(\mathbf{r}, \mathbf{r}^{\prime}\right) \sin \left(\phi-\phi^{\prime}\right) a d \phi^{\prime} d z^{\prime} \\
& \mp \int_{S_{c}} J_{r}\left(\mathbf{r}^{\prime}\right) G_{z^{\prime}}\left(\mathbf{r}, \mathbf{r}^{\prime}\right) \cos \left(\phi-\phi^{\prime}\right) \\
& +J_{\phi}\left(\mathbf{r}^{\prime}\right) G_{z^{\prime}}\left(\mathbf{r}, \mathbf{r}^{\prime}\right) \sin \left(\phi-\phi^{\prime}\right) r^{\prime} d \phi^{\prime} d r^{\prime} \\
& =\mathbf{u}_{r}(\mathbf{r}) \cdot\left[ \pm \mathbf{u}_{z} \times \mathbf{H}_{\mathrm{inc}}(\mathbf{r})\right] \\
& \frac{1}{2} J_{\phi}(\mathbf{r}) \pm \int_{S_{\mathrm{cs}}} J_{z}\left(\mathbf{r}^{\prime}\right)\left[G_{\phi^{\prime}}\left(\mathbf{r}, \mathbf{r}^{\prime}\right) \cos \left(\phi-\phi^{\prime}\right)\right. \\
& \left.-G_{r^{\prime}}\left(\mathbf{r}, \mathbf{r}^{\prime}\right) \sin \left(\phi-\phi^{\prime}\right)\right] a d \phi^{\prime} d z^{\prime} \\
& \mp \int_{S_{\mathrm{cs}}} J_{\phi}\left(\mathbf{r}^{\prime}\right) G_{z^{\prime}}\left(\mathbf{r}, \mathbf{r}^{\prime}\right) \cos \left(\phi-\phi^{\prime}\right) a d \phi^{\prime} d z^{\prime} \\
& \mp f_{S_{c}} J_{\phi}\left(\mathbf{r}^{\prime}\right) G_{z^{\prime}}\left(\mathbf{r}, \mathbf{r}^{\prime}\right) \cos \left(\phi-\phi^{\prime}\right) \\
& -J_{r}\left(\mathbf{r}^{\prime}\right) G_{z^{\prime}}\left(\mathbf{r}, \mathbf{r}^{\prime}\right) \sin \left(\phi-\phi^{\prime}\right) r^{\prime} d \phi^{\prime} d r^{\prime} \\
& \quad=\mathbf{u}_{\phi}(\mathbf{r}) \cdot\left[ \pm \mathbf{u}_{z} \times \mathbf{H}_{\mathrm{inc}}(\mathbf{r})\right] .
\end{aligned}
$$


Here, $f$ denotes the principal value [25] and $\mathbf{G}\left(\mathbf{r}, \mathbf{r}^{\prime}\right)=$ $\nabla^{\prime} g\left(\mathbf{r}, \mathbf{r}^{\prime}\right)=G_{r^{\prime}}\left(\mathbf{r}, \mathbf{r}^{\prime}\right) \mathbf{u}_{r^{\prime}}\left(\mathbf{r}^{\prime}\right)+G_{\phi^{\prime}}\left(\mathbf{r}, \mathbf{r}^{\prime}\right) \mathbf{u}_{\phi^{\prime}}\left(\mathbf{r}^{\prime}\right)+$ $G_{z^{\prime}}\left(\mathbf{r}, \mathbf{r}^{\prime}\right) \mathbf{u}_{z^{\prime}}\left(\mathbf{r}^{\prime}\right)$ with $g\left(\mathbf{r}, \mathbf{r}^{\prime}\right)$ the three-dimensional scalar Green's function in free-space. The surface current flowing on the cylindrical surface is $\mathbf{J}_{s}(\mathbf{r})=J_{z}(z, \phi) \mathbf{u}_{z}+J_{\phi}(z, \phi) \mathbf{u}_{\phi}(\mathbf{r})$ and the surface current on the caps is $\mathbf{J}_{s}(\mathbf{r})=J_{r}(r, \phi) \mathbf{u}_{r}(\mathbf{r})+$ $J_{\phi}(r, \phi) \mathbf{u}_{\phi}(\mathbf{r})$. The righthand term of these equations is determined by the incident field, which is caused by the magnetic surface current over the gap region in this specific configuration. Due to the rotational symmetry, we have no magnetic surface charge

$$
\nabla \cdot \mathbf{K}_{s}(\mathbf{r})=0
$$

So the incident magnetic field over the surface of the dipole is easily calculated [25]

$$
\mathbf{H}_{\mathrm{inc}}(\mathbf{r})=-\jmath \omega \epsilon_{0} \int_{S^{\prime}} \mathbf{K}_{s}\left(\mathbf{r}^{\prime}\right) g\left(\mathbf{r}, \mathbf{r}^{\prime}\right) d S^{\prime} .
$$

This equation leads to

$$
\begin{aligned}
& \mathbf{H}_{\mathrm{inc}}(\mathbf{r}) \cdot \mathbf{u}_{r}(\mathbf{r})=\frac{\jmath \omega \epsilon_{0}}{\delta_{g}} f_{S^{\prime}} \sin \left(\phi-\phi^{\prime}\right) g\left(\mathbf{r}, \mathbf{r}^{\prime}\right) d S^{\prime}=0 \\
& \mathbf{H}_{\mathrm{inc}}(\mathbf{r}) \cdot \mathbf{u}_{\phi}(\mathbf{r})=\frac{\jmath \omega \epsilon_{0}}{\delta_{g}} f_{S^{\prime}} \cos \left(\phi-\phi^{\prime}\right) g\left(\mathbf{r}, \mathbf{r}^{\prime}\right) d S^{\prime} \neq 0
\end{aligned}
$$

$$
\mathbf{H}_{\mathrm{inc}}(\mathbf{r}) \cdot \mathbf{u}_{z}(\mathbf{r}) .=0
$$

The incident field projected on $\mathbf{u}_{r}$ equals zero due to symmetry. Before starting to calculate the surface current distribution, it is interesting to verify whether there are no simplifications possible, due to the rotational symmetric configuration and symmetric source when the dipole is in free-space. Let us reconsider (4) and (5) with the source model as described above

$$
\begin{aligned}
& \frac{1}{2} J_{\phi}(\mathbf{r})-f_{S_{\mathrm{cs}}} J_{\phi}\left(\mathbf{r}^{\prime}\right) G_{r^{\prime}}\left(\mathbf{r}, \mathbf{r}^{\prime}\right) a d \phi^{\prime} d z^{\prime} \\
& +\int_{S_{c}} J_{r}\left(\mathbf{r}^{\prime}\right) G_{\phi^{\prime}}\left(\mathbf{r}, \mathbf{r}^{\prime}\right)-J_{\phi}\left(\mathbf{r}^{\prime}\right) G_{r^{\prime}}\left(\mathbf{r}, \mathbf{r}^{\prime}\right) r^{\prime} d \phi^{\prime} d r^{\prime} \\
& \quad=\mathbf{u}_{\phi}(\mathbf{r}) \cdot\left[\mathbf{u}_{r}(\mathbf{r}) \times \mathbf{H}_{\mathrm{inc}}(\mathbf{r})\right]=0 \\
& \frac{1}{2} J_{\phi}(\mathbf{r}) \pm \int_{S_{\mathrm{cs}}} J_{z}\left(\mathbf{r}^{\prime}\right)\left[G_{\phi^{\prime}}\left(\mathbf{r}, \mathbf{r}^{\prime}\right) \cos \left(\phi-\phi^{\prime}\right)\right. \\
& \left.-G_{r^{\prime}}\left(\mathbf{r}, \mathbf{r}^{\prime}\right) \sin \left(\phi-\phi^{\prime}\right)\right] a d \phi^{\prime} d z^{\prime} \\
& \mp \int_{S_{\mathrm{cs}}} J_{\phi}\left(\mathbf{r}^{\prime}\right) G_{z^{\prime}}\left(\mathbf{r}, \mathbf{r}^{\prime}\right) \cos \left(\phi-\phi^{\prime}\right) a d \phi^{\prime} d z^{\prime} \\
& \mp \underset{\int_{c}}{f_{\phi}} J_{\phi}\left(\mathbf{r}^{\prime}\right) G_{z^{\prime}}\left(\mathbf{r}, \mathbf{r}^{\prime}\right) \cos \left(\phi-\phi^{\prime}\right) \\
& -J_{r}\left(\mathbf{r}^{\prime}\right) G_{z^{\prime}}\left(\mathbf{r}, \mathbf{r}^{\prime}\right) \sin \left(\phi-\phi^{\prime}\right) r^{\prime} d \phi^{\prime} d r^{\prime} \\
& =\mathbf{u}_{\phi}(\mathbf{r}) \cdot\left[ \pm \mathbf{u}_{z} \times \mathbf{H}_{\mathrm{inc}}(\mathbf{r})\right]=0 .
\end{aligned}
$$

With regard to the expressions of kernels, we can write

$$
\begin{aligned}
& G_{r^{\prime}}\left(\mathbf{r}, \mathbf{r}^{\prime}\right)=f\left(\left|\mathbf{r}-\mathbf{r}^{\prime}\right|\right) \cdot\left(r^{\prime}-r \cos \left(\phi-\phi^{\prime}\right)\right) \\
& \text { Even in } \phi^{\prime} \text { around } \phi \\
& G_{\phi^{\prime}}\left(\mathbf{r}, \mathbf{r}^{\prime}\right)=-f\left(\left|\mathbf{r}-\mathbf{r}^{\prime}\right|\right) \cdot r \sin \left(\phi-\phi^{\prime}\right) \\
& \text { Odd in } \phi^{\prime} \text { around } \phi \\
& G_{z^{\prime}}\left(\mathbf{r}, \mathbf{r}^{\prime}\right)=-f\left(\left|\mathbf{r}-\mathbf{r}^{\prime}\right|\right) \cdot\left(z-z^{\prime}\right) \\
& \text { Even in } \phi^{\prime} \text { around } \phi .
\end{aligned}
$$

Every surface current distribution must be rotational invariant, due to the rotational symmetry of the configuration and source. This means that the surface currents can be placed outside the integration over $\phi^{\prime}$. Integrating odd functions over a symmetric interval gives zero, while integrating an even function over a symmetric interval will be different from zero. Therefore, (12) and (13) can be written as

$$
\begin{aligned}
& \frac{1}{2} J_{\phi}(\mathbf{r})-\int_{S_{\mathrm{cs}}} J_{\phi}\left(\mathbf{r}^{\prime}\right) G_{r^{\prime}}\left(\mathbf{r}, \mathbf{r}^{\prime}\right) a d \phi^{\prime} d z^{\prime} \\
& -\int_{S_{c}} J_{\phi}\left(\mathbf{r}^{\prime}\right) G_{r^{\prime}}\left(\mathbf{r}, \mathbf{r}^{\prime}\right) r^{\prime} d \phi^{\prime} d r^{\prime}=0 \\
& \frac{1}{2} J_{\phi}(\mathbf{r}) \mp \int_{S_{\mathrm{cs}}} J_{\phi}\left(\mathbf{r}^{\prime}\right) G_{z^{\prime}}\left(\mathbf{r}, \mathbf{r}^{\prime}\right) \cos \left(\phi-\phi^{\prime}\right) a d \phi^{\prime} d z^{\prime} \\
& \mp \underset{S_{c}}{ } J_{\phi}\left(\mathbf{r}^{\prime}\right) G_{z^{\prime}}\left(\mathbf{r}, \mathbf{r}^{\prime}\right) \cos \left(\phi-\phi^{\prime}\right) r^{\prime} d \phi^{\prime} d r^{\prime}=0 .
\end{aligned}
$$

Unless these coupled integral equations have eigenvalues equal to zero, we can conclude that $J_{\phi}(\mathbf{r})=0$ for all $\mathbf{r}$. Imposing this condition reduces strongly the size of the interaction matrix. If one calculates the surface current distribution without imposing $J_{\phi}(\mathbf{r})=0$, the calculated value of $J_{\phi}(\mathbf{r})$ is equal to the numerical noise.

\section{B. Results}

We first compare our results with results from the commercial electromagnetic software package NEC [28]. The basis functions and test procedure we apply are identical as described in a previous publication [29]. The NEC code is very fast but also has a number of limitations, which are not present in our method. A first limitation is with regard to the segment length (a segment is the interval on the dipole between two point-match points). The segment length should be smaller than $0.1 \lambda$. This is a reasonable condition since the field can change significantly over this distance. For the wire radius, it is stated that $2 \pi a / \lambda$ should be much less than 1 . This condition excludes thicker wires. An upper limit on the number of point-match points is imposed by the condition that the ratio of the segment length over the radius $a$ should be greater than 0.5 . Further, the gap height $\delta_{g}$ is equal to the segment length. An antenna with a small gap (relative to the radius $a$ ) can thus not correctly be modeled by use of NEC. In the method we use, the size of the gap is independent of the number of point-match locations and there are no limitations with regard to the radius. 


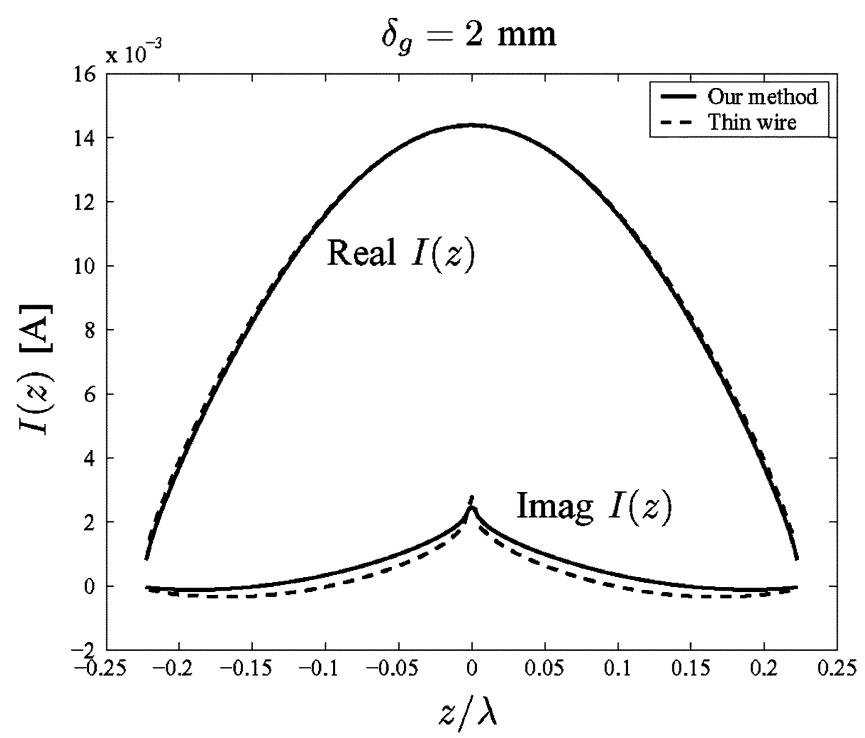

Fig. 2. Distribution of the equivalent line current with our method and NEC for a dipole with $h / \lambda=0.447, a / \lambda=5.4 \cdot 10^{-3}$, and $\delta_{g} / \lambda=6 \cdot 10^{-3}$.

A first example we treat is a dipole antenna we will use later in the experimental verification, namely an antenna with radius $a=1.8 \mathrm{~mm}$ at $900 \mathrm{MHz}$ (thus $a \approx \lambda / 185$ ), a length $h$ of $149 \mathrm{~mm}(\approx \lambda / 2.24)$ and $\delta_{g}=1 \mathrm{~mm}(\approx \lambda / 333)$. If we use NEC for this gap height, numerical difficulties occur (as explained further). So only in order to compare our method with NEC, we choose $\delta_{g}$ as $2 \mathrm{~mm}$. To obtain the equivalent line current, we have to integrate $J_{z}(z, \phi)$ over the circumference of the dipole, as defined by (19)

$$
I(z)=\int_{0}^{2 \pi} J_{z}(z, \phi) a d \phi .
$$

On Fig. 2 the real and imaginary parts of the equivalent line current are plotted for the dipole under study. The real part of the equivalent line current corresponds very well. The imaginary part is slightly different. This is due to the different source model. In NEC, the incident electric field is set to $1 / \delta_{g}$ at the central segment and zero at all other point-match points. In our method, we calculate the incident magnetic field at all pointmatch locations. As mentioned previously, the gap height for NEC is limited at the lower end by the condition that $\delta_{g} / a>$ 0.5 . If we take $\delta_{g}=1 \mathrm{~mm}$ in the previous dipole, we obtain that $\delta_{g} / a \approx 0.55$, which fulfills the previous condition. But for the imaginary part of the current, anomalies occur. If the height of the gap is further reduced to e.g., $0.75 \mathrm{~mm}\left(\delta_{g} / a \approx 0.42\right)$, the imaginary current component of the thin-wire approximation becomes unstable at the center of the dipole and the real part at the dipole ends, in contrast to our method, shown on Fig. 3.

As a final numerical validation, we can calculate the total magnetic field in and outside the dipole antenna. For any location $\mathbf{r},(20)$ is valid

$$
\mathbf{H}_{\mathrm{sc}}(\mathbf{r})=\int_{S^{\prime}} \mathbf{J}_{s}\left(\mathbf{r}^{\prime}\right) \times \mathbf{G}\left(\mathbf{r}, \mathbf{r}^{\prime}\right) d S^{\prime}
$$

When $\mathbf{r}$ is positioned on the PEC surface, a principal value and self-patch are defined [25]. For all other locations, no numerical

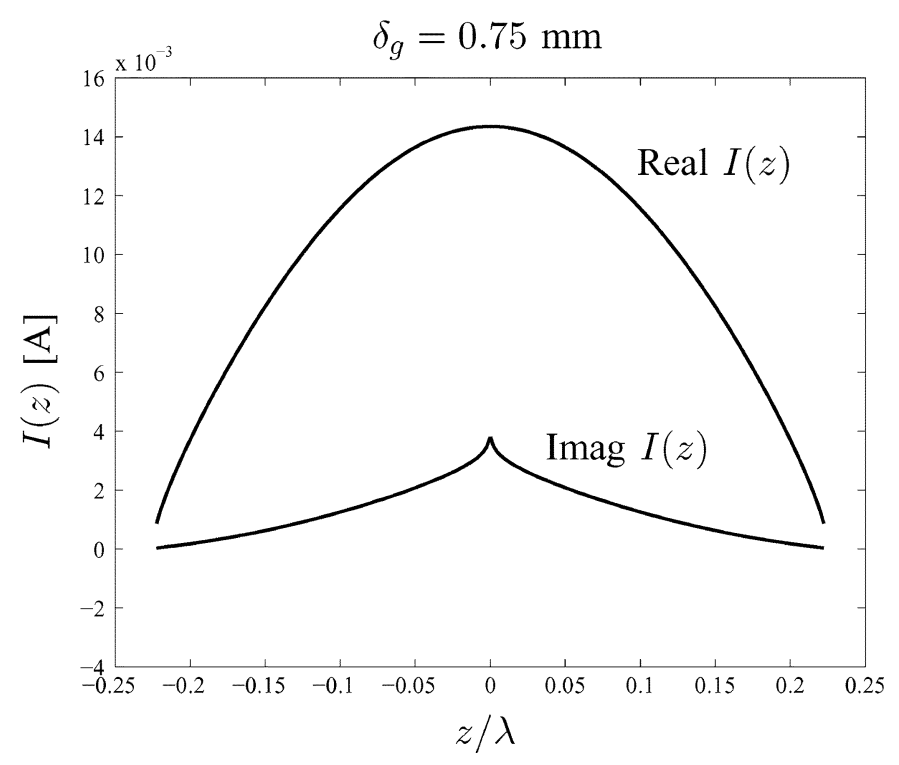

Fig. 3. Distribution of the equivalent line current calculated with our method for $\delta_{g}=0.75 \mathrm{~mm}$.

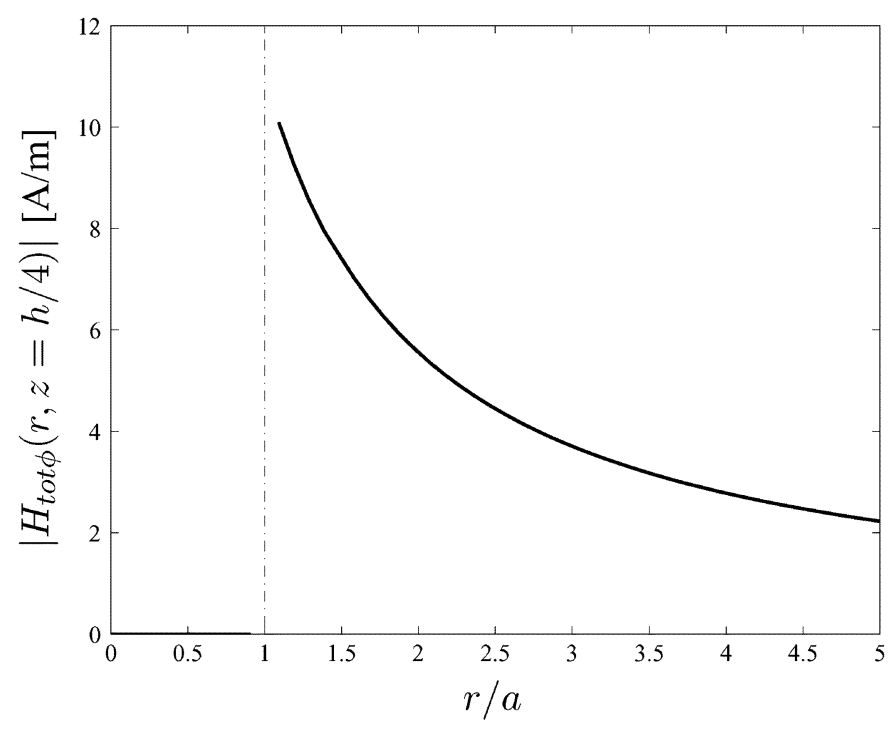

Fig. 4. Radial variation of the total field.

difficulties occur in the evaluation of the integral. Based on the fact that $J_{\phi}\left(\mathbf{r}^{\prime}\right)$ equals zero for all $\mathbf{r}^{\prime}$, that all other surface current components are rotational symmetric and that $G_{\phi^{\prime}}$ has an odd dependency in $\phi^{\prime}$, it is clear that $H_{\mathrm{sc} r}$ and $H_{\mathrm{sc} z}$ are zero and only $H_{\mathrm{sc} \phi}$ is different from zero. Since the incident field from the excitation also has only a $\phi$-component, we also find that the total magnetic field has only one cylindrical component different from zero, namely $H_{\text {tot } \phi}$. We have plotted this component for $r$ going from zero to $5 a$ and $z=h / 4$ in Fig. 4 . The parameters of the antenna are again $h=0.447 \lambda, a=$ $\lambda / 185$, and $\delta_{g}=1 \mathrm{~mm}$. The values are normalized to a radiated power of $1 \mathrm{~W}$. We approach the PEC surface as close as $\lambda / 2000$. The calculated field is indeed zero inside the PEC body, which again confirms the correctness of our model. Finally, we show the magnitude of the distribution of the normalized total field $H_{\text {tot } n}(z, r)=H_{\text {tot } \phi}(z, r) / \max \left(H_{\text {tot } \phi}(z, r)\right)$ close to the antenna. We have plotted $20 \log \left|H_{\text {totn }}(z, r)\right|$ in Fig. 5. 


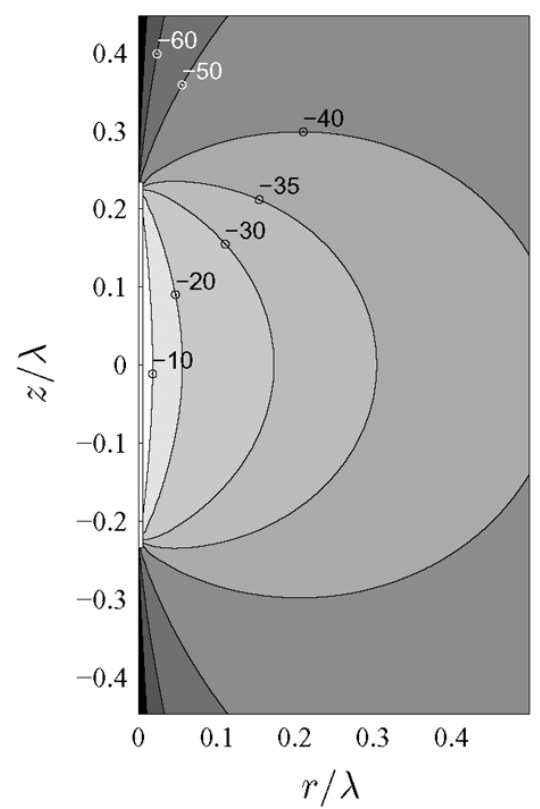

Fig. 5. Variation of the normalized total magnetic field [dB].

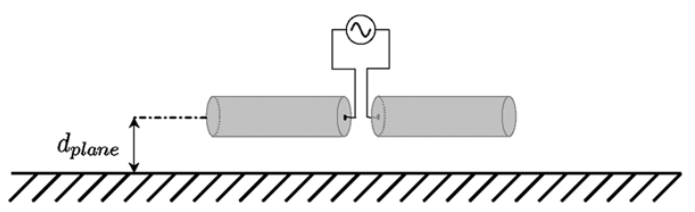

Fig. 6. Dipole near an infinite PEC plane.

\section{Dipole NeAR A CONDUCTING SCREEN}

When the dipole is located near a conducting object, the current density distribution will become nonrotational symmetric. In this section, we will describe the behavior of a dipole near an infinite perfectly electrical conducting (PEC) plane, as shown in Fig. 6. We have done this study as it can be considered as a worst case configuration of a dipole antenna near a conducting object. It is demonstrated that the conducting plate has a nonnegligible impact on the surface current distribution, especially if the electric distance is small.

\section{A. Construction of the Interaction Matrix}

The solution method is based on the modification of the kernel of the original field integral equation. The configuration of Fig. 6 is best modeled by use of image theory [26]. At the boundary of the conducting plane, the normal component of the magnetic field and the tangential component of the electric field must vanish. This is accomplished by placing an image current distribution symmetric with respect to the boundary. So the original configuration of Fig. 6 can be replaced by two aligned dipoles at a distance of $d=2 d_{\text {plane }}$ from each other, with the condition of the image theory imposed on the surface currents of the mirror dipole. In order to take the mirror dipole into account in the MFIE, we have to calculate the scattered field at the real dipole surface due to the image surface currents

$$
\begin{aligned}
\mathbf{J}_{s}(\mathbf{r}) & =\mathbf{u}_{\mathbf{n}}(\mathbf{r}) \times \mathbf{H}_{\mathrm{tot}}(\mathbf{r}) \\
& =\mathbf{u}_{\mathbf{n}}(\mathbf{r}) \times \mathbf{H}_{\mathrm{inc}}(\mathbf{r})+\mathbf{u}_{\mathbf{n}}(\mathbf{r}) \times \mathbf{H}_{\mathrm{sc}}(\mathbf{r}) .
\end{aligned}
$$

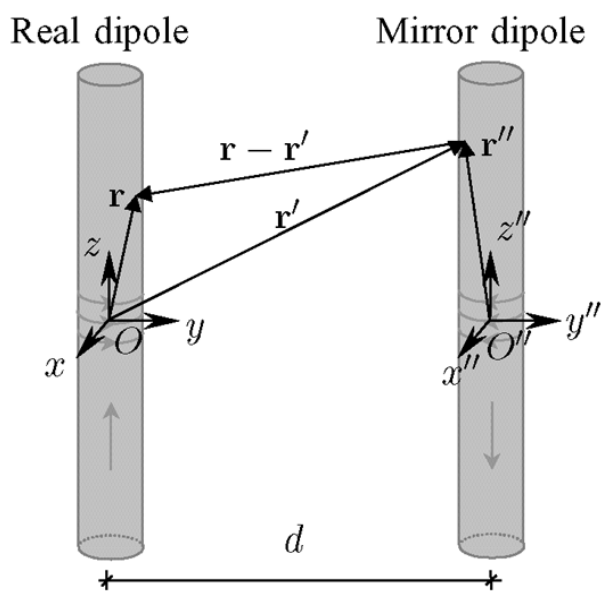

Fig. 7. Used conventions for the calculation of the scattered field due to the image dipole.

The scattered field now consists of four terms. The first two terms are the self-patch and the field caused by the electrical surface currents at all other locations of the real dipole. These terms have been formulated for the dipole in free-space configuration. The additional terms are due to the electric and magnetic image surface currents. Let us call these fields respectively $\mathbf{H}_{\mathrm{sc}_{\mathrm{im} J}}(\mathbf{r})$ and $\mathbf{H}_{\mathrm{sc}_{\mathrm{imK}}}(\mathbf{r})$. For the calculation of the scattered field, we use the notations and conventions as indicated on Fig. 7. The first part of the scattered magnetic field we will calculate is the field due to the electric image surface currents. Following the notations of Fig. 7, we can write that

$$
\mathbf{H}_{\mathrm{sc}_{\mathrm{im}} \mathrm{J}}(\mathbf{r})=\int_{S^{\prime}} \mathbf{J}_{s_{\mathrm{im}}}\left(\mathbf{r}^{\prime}\right) \times \mathbf{G}\left(\mathbf{r}, \mathbf{r}^{\prime}\right) d S^{\prime}
$$

where $S^{\prime}$ is the surface of the mirror dipole and $\mathbf{J}_{s_{\text {im }}}$ is the electric surface current distribution on that dipole. At this stage, we do not yet enforce the image surface current conditions. Based on the image conditions and the invariance of the cartesian unit vectors under translation, it is better to write the kernel of the integral in cartesian coordinates. For the integration itself, it is much easier to use $\left(x^{\prime \prime}, y^{\prime \prime}, z^{\prime \prime}\right)$ as the reference coordinate system. $\mathbf{r}^{\prime}$ and $\mathbf{r}^{\prime \prime}$ indicate the same spatial location and are both describing the surface of the image dipole (noted as $S^{\prime}$ or $S^{\prime \prime}$ ), but with another reference coordinate system $O^{\prime}$ and $O^{\prime \prime}$ respectively. One can write that

$$
\mathbf{G}\left(\mathbf{r}, \mathbf{r}^{\prime}\right)=\frac{1}{4 \pi} \nabla^{\prime}\left(\frac{e^{-\jmath k_{0}\left|\mathbf{r}-\mathbf{r}^{\prime}\right|}}{\left|\mathbf{r}-\mathbf{r}^{\prime}\right|}\right)=\sum_{i=x, y, z} G_{i^{\prime}}\left(\mathbf{r}, \mathbf{r}^{\prime}\right) \mathbf{u}_{i^{\prime}}
$$

with

$$
G_{i^{\prime}}\left(\mathbf{r}, \mathbf{r}^{\prime}\right)=-f\left(\left|\mathbf{r}-\mathbf{r}^{\prime}\right|\right)\left(i-i^{\prime}\right) \quad \text { with } i=x, y, z
$$

and

$$
f\left(\left|\mathbf{r}-\mathbf{r}^{\prime}\right|\right)=\frac{g\left(\mathbf{r}, \mathbf{r}^{\prime}\right)}{\left|\mathbf{r}-\mathbf{r}^{\prime}\right|}\left(-\jmath k_{0}-\frac{1}{\left|\mathbf{r}-\mathbf{r}^{\prime}\right|}\right) .
$$

Fig. 7 shows that $\mathbf{r}^{\prime}=\mathbf{r}^{\prime \prime}+d \cdot \mathbf{u}_{y}$, which leads to an expression of $\mathbf{G}$ in the local coordinate system $\left(x^{\prime \prime}, y^{\prime \prime}, z^{\prime \prime}\right)$ of the image 
dipole in cylindrical coordinates

$$
\begin{aligned}
G_{x^{\prime \prime}}\left(\mathbf{r}, \mathbf{r}^{\prime \prime}\right)= & -f\left(\left|\mathbf{r}-\mathbf{r}^{\prime \prime}-d \mathbf{u}_{y}\right|\right) \\
& \times\left(r \cos \phi-r^{\prime \prime} \cos \phi^{\prime \prime}\right)(-1) \\
G_{y^{\prime \prime}}\left(\mathbf{r}, \mathbf{r}^{\prime \prime}\right)= & -f\left(\left|\mathbf{r}-\mathbf{r}^{\prime \prime}-d \mathbf{u}_{y}\right|\right) \\
& \times\left(r \sin \phi-r^{\prime \prime} \sin \phi^{\prime \prime}-d\right)(-1) \\
G_{z^{\prime \prime}}\left(\mathbf{r}, \mathbf{r}^{\prime \prime}\right)= & -f\left(\left|\mathbf{r}-\mathbf{r}^{\prime \prime}-d \mathbf{u}_{y}\right|\right)\left(z-z^{\prime \prime}\right)(-1)
\end{aligned}
$$

where

$$
\begin{array}{r}
\left|\mathbf{r}-\mathbf{r}^{\prime \prime}-d \mathbf{u}_{y}\right|=\left[r^{2}+r^{\prime \prime 2}-2 r r^{\prime \prime} \cos \left(\phi-\phi^{\prime \prime}\right)+\left(z-z^{\prime \prime}\right)^{2}\right. \\
\left.-2 d\left(r \sin \phi-r^{\prime \prime} \sin \phi^{\prime \prime}\right)+d^{2}\right]^{1 / 2} .
\end{array}
$$

Using these manipulations, we have written the scattered field (generated by the surface currents flowing on the mirror dipole) on the real dipole at $O$ as an integral over the local coordinates at $O^{\prime \prime}$

$$
\mathbf{H}_{\mathrm{sc}_{\mathrm{imJ}}}(\mathbf{r})=\int_{S^{\prime \prime}} \mathbf{J}_{s_{\mathrm{im}}}\left(\mathbf{r}^{\prime \prime}\right) \times \mathbf{G}\left(\mathbf{r}, \mathbf{r}^{\prime \prime}\right) d S^{\prime \prime}
$$

Now, we can make use of the image relationships. When we elaborate the kernel of (28), we obtain

$$
\begin{aligned}
\mathbf{J}_{s_{\mathrm{im}}}\left(\mathbf{r}^{\prime \prime}\right) \times \mathbf{G}\left(\mathbf{r}, \mathbf{r}^{\prime \prime}\right) & \\
= & \mathbf{u}_{x}\left[J_{s_{\mathrm{im}} y}\left(\mathbf{r}^{\prime \prime}\right) G_{z}\left(\mathbf{r}, \mathbf{r}^{\prime \prime}\right)-J_{s_{\mathrm{im}} z}\left(\mathbf{r}^{\prime \prime}\right) G_{y}\left(\mathbf{r}, \mathbf{r}^{\prime \prime}\right)\right] \\
& +\mathbf{u}_{y}\left[J_{s_{\mathrm{im}} z}\left(\mathbf{r}^{\prime \prime}\right) G_{x}\left(\mathbf{r}, \mathbf{r}^{\prime \prime}\right)-J_{s_{\mathrm{im}} x}\left(\mathbf{r}^{\prime \prime}\right) G_{z}\left(\mathbf{r}, \mathbf{r}^{\prime \prime}\right)\right] \\
& +\mathbf{u}_{z}\left[J_{s_{\mathrm{im}} x}\left(\mathbf{r}^{\prime \prime}\right) G_{y}\left(\mathbf{r}, \mathbf{r}^{\prime \prime}\right)-J_{s_{\mathrm{im}} y}\left(\mathbf{r}^{\prime \prime}\right) G_{x}\left(\mathbf{r}, \mathbf{r}^{\prime \prime}\right)\right] .
\end{aligned}
$$

We remark that we can write $G_{i}\left(\mathbf{r}, \mathbf{r}^{\prime \prime}\right)$ instead of $G_{i}^{\prime \prime}\left(\mathbf{r}, \mathbf{r}^{\prime \prime}\right)$ since $\mathbf{u}_{i^{\prime \prime}}=\mathbf{u}_{i}(i=x, y, z)$ due to the invariance of cartesian unit vectors under translation. Let us call the surface current densities on the real dipole $\mathbf{J}_{s}$. We know that the current densities on the image dipole are identical to those on the real dipole in magnitude but the phase is modified according to the image theory. This leads to

$$
\begin{aligned}
\mathbf{J}_{s_{\mathrm{im}}}\left(\mathbf{r}^{\prime \prime}\right) \times \mathbf{G}\left(\mathbf{r}, \mathbf{r}^{\prime \prime}\right) & \\
= & \mathbf{u}_{x}\left[J_{s y}\left(\mathbf{r}^{\prime \prime}\right) G_{z}\left(\mathbf{r}, \mathbf{r}^{\prime \prime}\right)+J_{s z}\left(\mathbf{r}^{\prime \prime}\right) G_{y}\left(\mathbf{r}, \mathbf{r}^{\prime \prime}\right)\right] \\
& +\mathbf{u}_{y}\left[J_{s x}\left(\mathbf{r}^{\prime \prime}\right) G_{z}\left(\mathbf{r}, \mathbf{r}^{\prime \prime}\right)-J_{s z}\left(\mathbf{r}^{\prime \prime}\right) G_{x}\left(\mathbf{r}, \mathbf{r}^{\prime \prime}\right)\right] \\
& +\mathbf{u}_{z}\left[-J_{s x}\left(\mathbf{r}^{\prime \prime}\right) G_{y}\left(\mathbf{r}, \mathbf{r}^{\prime \prime}\right)-J_{s y}\left(\mathbf{r}^{\prime \prime}\right) G_{x}\left(\mathbf{r}, \mathbf{r}^{\prime \prime}\right)\right]
\end{aligned}
$$

In order to use the scattered field in the MoM, we need to project $\mathbf{u}_{\mathbf{n}}(\mathbf{r}) \times \mathbf{H}_{\mathrm{sc}_{\mathrm{imJ}}}(\mathbf{r})$ on the tangential directions of the surface of the real dipole. For the observation point $\mathbf{r}$ located on the cylindrical surface, we have [ $S_{\mathrm{cS}}^{\prime \prime}$ and $S_{c}^{\prime \prime}$ stand, respectively, for the cylindrical surface and cap surfaces, as in (3) to (6)]

$$
\begin{aligned}
\mathbf{u}_{z} \cdot & {\left[\mathbf{u}_{r} \times \mathbf{H}_{\mathrm{sC}_{\mathrm{imJ}}}(\mathbf{r})\right] } \\
= & \int_{S_{\mathrm{cs}}^{\prime \prime}} J_{z}\left(\mathbf{r}^{\prime \prime}\right)\left[-G_{x}\left(\mathbf{r}, \mathbf{r}^{\prime \prime}\right) \cos \phi\right. \\
& \left.-G_{y}\left(\mathbf{r}, \mathbf{r}^{\prime \prime}\right) \sin \phi\right] a d \phi^{\prime \prime} d z^{\prime \prime} \\
& +\int_{S_{\mathrm{cs}}^{\prime \prime}} J_{\phi}\left(\mathbf{r}^{\prime \prime}\right)\left[-G_{z}\left(\mathbf{r}, \mathbf{r}^{\prime \prime}\right) \sin \left(\phi+\phi^{\prime \prime}\right)\right] a d \phi^{\prime \prime} d z^{\prime \prime} \\
& +\int_{S_{c}^{\prime \prime}} J_{r}\left(\mathbf{r}^{\prime \prime}\right) G_{z}\left(\mathbf{r}, \mathbf{r}^{\prime \prime}\right) \cos \left(\phi+\phi^{\prime \prime}\right) r^{\prime \prime} d \phi^{\prime \prime} d r^{\prime \prime} \\
& +\int_{S_{c}^{\prime \prime}} J_{\phi}\left(\mathbf{r}^{\prime \prime}\right)\left[-G_{z}\left(\mathbf{r}, \mathbf{r}^{\prime \prime}\right) \sin \left(\phi+\phi^{\prime \prime}\right)\right] r^{\prime \prime} d \phi^{\prime \prime} d r^{\prime \prime}
\end{aligned}
$$

$$
\begin{aligned}
\mathbf{u}_{\phi} \cdot & {\left[\mathbf{u}_{r} \times \mathbf{H}_{\mathrm{sc}_{\mathrm{im}} \mathrm{J}}(\mathbf{r})\right] } \\
= & \int_{S_{\mathrm{cs}}^{\prime \prime}} J_{\phi}\left(\mathbf{r}^{\prime \prime}\right)\left[G_{x}\left(\mathbf{r}, \mathbf{r}^{\prime \prime}\right) \cos \phi^{\prime \prime}\right. \\
& \left.-G_{y}\left(\mathbf{r}, \mathbf{r}^{\prime \prime}\right) \sin \phi^{\prime \prime}\right] a d \phi^{\prime \prime} d z^{\prime \prime} \\
& +\int_{S_{c}^{\prime \prime}} J_{r}\left(\mathbf{r}^{\prime \prime}\right)\left[G_{x}\left(\mathbf{r}, \mathbf{r}^{\prime \prime}\right) \sin \phi^{\prime \prime}\right. \\
& \left.+G_{y}\left(\mathbf{r}, \mathbf{r}^{\prime \prime}\right) \cos \phi^{\prime \prime}\right] r^{\prime \prime} d \phi^{\prime \prime} d r^{\prime \prime} \\
& +\int_{S_{c}^{\prime \prime}} J_{\phi}\left(\mathbf{r}^{\prime \prime}\right)\left[G_{x}\left(\mathbf{r}, \mathbf{r}^{\prime \prime}\right) \cos \phi^{\prime \prime}\right. \\
& \left.-G_{y}\left(\mathbf{r}, \mathbf{r}^{\prime \prime}\right) \sin \phi^{\prime \prime}\right] r^{\prime \prime} d \phi^{\prime \prime} d r^{\prime \prime} .
\end{aligned}
$$

For the observation point located on the caps, we can write

$$
\begin{aligned}
\mathbf{u}_{r} \cdot & {\left[ \pm \mathbf{u}_{z} \times \mathbf{H}_{\mathrm{scimJ}}(\mathbf{r})\right] } \\
= & \pm \int_{S_{\mathrm{cs}}^{\prime \prime}} J_{z}\left(\mathbf{r}^{\prime \prime}\right)\left[G_{x}\left(\mathbf{r}, \mathbf{r}^{\prime \prime}\right) \cos \phi\right. \\
& \left.+G_{y}\left(\mathbf{r}, \mathbf{r}^{\prime \prime}\right) \sin \phi\right] a d \phi^{\prime \prime} d z^{\prime \prime} \\
& \pm \int_{S_{\mathrm{cs}}^{\prime \prime}} J_{\phi}\left(\mathbf{r}^{\prime \prime}\right) G_{z}\left(\mathbf{r}, \mathbf{r}^{\prime \prime}\right) \sin \left(\phi+\phi^{\prime \prime}\right) a d \phi^{\prime \prime} d z^{\prime \prime} \\
& \mp \int_{S_{c}^{\prime \prime}} J_{r}\left(\mathbf{r}^{\prime \prime}\right) G_{z}\left(\mathbf{r}, \mathbf{r}^{\prime \prime}\right) \cos \left(\phi+\phi^{\prime \prime}\right) r^{\prime \prime} d \phi^{\prime \prime} d r^{\prime \prime} \\
& \pm \int_{S_{c}^{\prime \prime}} J_{\phi}\left(\mathbf{r}^{\prime \prime}\right) G_{z}\left(\mathbf{r}, \mathbf{r}^{\prime \prime}\right) \sin \left(\phi+\phi^{\prime \prime}\right) r^{\prime \prime} d \phi^{\prime \prime} d r^{\prime \prime} \\
\mathbf{u}_{\phi} \cdot & {\left[ \pm \mathbf{u}_{z} \times \mathbf{H}_{\mathrm{sc}_{\mathrm{im}} \mathrm{J}}(\mathbf{r})\right] } \\
= & \pm \int_{S_{\mathrm{cs}}^{\prime \prime}} J_{z}\left(\mathbf{r}^{\prime \prime}\right)\left[G_{x}\left(\mathbf{r}, \mathbf{r}^{\prime \prime}\right) \sin \phi\right. \\
& \left.-G_{y}\left(\mathbf{r}, \mathbf{r}^{\prime \prime}\right) \cos \phi\right] a d \phi^{\prime \prime} d z^{\prime \prime} \\
& \mp \int_{S_{\mathrm{cs}}^{\prime \prime}} J_{\phi}\left(\mathbf{r}^{\prime \prime}\right) G_{z}\left(\mathbf{r}, \mathbf{r}^{\prime \prime}\right) \cos \left(\phi+\phi^{\prime \prime}\right) a d \phi^{\prime \prime} d z^{\prime \prime} \\
& \mp \int_{S_{c}^{\prime \prime}} J_{r}\left(\mathbf{r}^{\prime \prime}\right) G_{z}\left(\mathbf{r}, \mathbf{r}^{\prime \prime}\right) \sin \left(\phi+\phi^{\prime \prime}\right) r^{\prime \prime} d \phi^{\prime \prime} d r^{\prime \prime} \\
& \mp \int_{S_{c}^{\prime \prime}} J_{\phi}\left(\mathbf{r}^{\prime \prime}\right) G_{z}\left(\mathbf{r}, \mathbf{r}^{\prime \prime}\right) \cos \left(\phi+\phi^{\prime \prime}\right) r^{\prime \prime} d \phi^{\prime \prime} d r^{\prime \prime} .(34)
\end{aligned}
$$

These equations are used to construct an interaction matrix by the method of moments that takes into account the reflection of the electric surface currents on the PEC plane. In order to obtain the total interaction matrix, one simply has to add the mirror interaction matrix to the one of the dipole in free-space. When the distance $d_{\text {plane }}$ is modified, the only interaction matrix that has to be calculated is the mirror matrix; the free-space interaction matrix remains of course unchanged.

Not only an electric surface current flows on the mirror dipole, but also a magnetic surface current $\mathbf{K}_{s_{\text {im }}}$ flows on the mirror surface, corresponding to the gap region of the real dipole. Based on image theory, we have (for $\mathbf{r}^{\prime \prime}$ in the gap region)

$$
\mathbf{K}_{s_{\mathrm{im}}}\left(\mathbf{r}^{\prime \prime}\right)=\frac{1}{\delta_{g}} \mathbf{u}_{\phi^{\prime \prime}}\left(\mathbf{r}^{\prime \prime}\right) .
$$

The scattered field caused by the image of the original source is ( $S^{\prime \prime}$ is the surface of the gap region)

$$
\mathbf{H}_{\mathrm{sc}_{\mathrm{imK}}}(\mathbf{r})=-\jmath \omega \epsilon_{0} \int_{S^{\prime \prime}} \mathbf{K}_{s_{\mathrm{im}}}\left(\mathbf{r}^{\prime \prime}\right) g\left(\mathbf{r}, \mathbf{r}^{\prime \prime}-d \mathbf{u}_{y}\right) d S^{\prime \prime}
$$




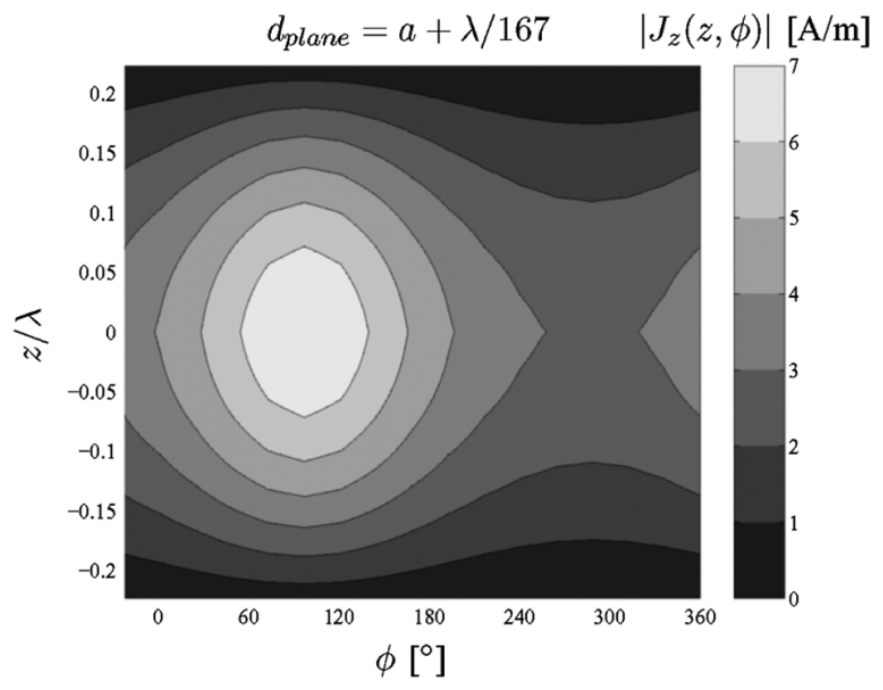

Fig. 8. Distribution of $\left|J_{z}(z, \phi)\right|$ over the cylindrical surface for $d_{\text {plane }}=$ $a+\lambda / 167$.

Projection on the cylindrical coordinates of the real dipole gives

$$
\begin{aligned}
& \mathbf{H}_{\mathrm{sC}_{\mathrm{imK}}}(\mathbf{r}) \cdot \mathbf{u}_{r}(\mathbf{r}) \\
& \quad=-\frac{\jmath \omega \epsilon_{0}}{\delta_{g}} \int_{S^{\prime \prime}} \sin \left(\phi-\phi^{\prime \prime}\right) g\left(\mathbf{r}, \mathbf{r}^{\prime \prime}-d \mathbf{u}_{y}\right) d S^{\prime \prime} \neq 0 \\
& \mathbf{H}_{\mathrm{sC}_{\mathrm{imK}}}(\mathbf{r}) \cdot \mathbf{u}_{\phi}(\mathbf{r}) \\
& \quad=-\frac{\jmath \omega \epsilon_{0}}{\delta_{g}} \int_{S^{\prime \prime}} \cos \left(\phi-\phi^{\prime}\right) g\left(\mathbf{r}, \mathbf{r}^{\prime \prime}-d \mathbf{u}_{y}\right) d S^{\prime \prime} \neq 0 \\
& \mathbf{H}_{\mathrm{SC}_{\mathrm{imK}}}(\mathbf{r}) \cdot \mathbf{u}_{z}(\mathbf{r})=0 .
\end{aligned}
$$

We remark that $\mathbf{H}_{\mathrm{Sc}_{\mathrm{imK}}}(\mathbf{r}) \cdot \mathbf{u}_{r}(\mathbf{r})$ is not equal to zero anymore since, for $d \neq 0, g\left(\mathbf{r}, \mathbf{r}^{\prime}\right)$ is not symmetric in $\phi . \mathbf{H}_{\mathrm{sc}_{\mathrm{imK}}}$ is independent of $\mathbf{J}_{s}$ so it can be considered as an additional incident field in the field integral equation.

\section{B. Results}

We have again taken the antenna from previous sections as an example, namely $h=\lambda / 2.24, a=\lambda / 185$, and $\delta_{g}=\lambda / 333$. Fig. 8 shows the distribution of $\left|J_{z}(z, \phi)\right|$ over the cylindrical surface. At $900 \mathrm{MHz}$, the distance $d_{\text {plane }}-a$ equals $2 \mathrm{~mm}$. The presence of the plane is clearly introducing a strong $\phi$-dependency of the current distribution. On Figs. 9 and 10, the $\left|J_{z}(z, \phi)\right|$ is shown for larger distances. The coupling between the dipole and plane clearly decreases as $d_{\text {plane }}$ increases, as could be expected from a physical point of view. The results of the measurement and the modeled admittance as a function of the distance between the dipole and the plane can be seen on Figs. 11 and 12, which shows excellent agreement. The measured admittance at the gap $Y_{\delta_{g}}=G_{\delta_{g}}+\jmath B_{\delta_{g}}$ was obtained by a reflection measurement with a network analyzer (NWA) and applying a de-embedding procedure on the time domain reflection response in order to derive the admittance at the gap. The size of the rectangular plane in our measurement set-up was $1.8 \lambda$ by $3 \lambda$.

Finally, we investigate the influence of imposing $J_{\phi}(z, \phi)=$ 0 since this reduces the size of the interaction matrix. From a

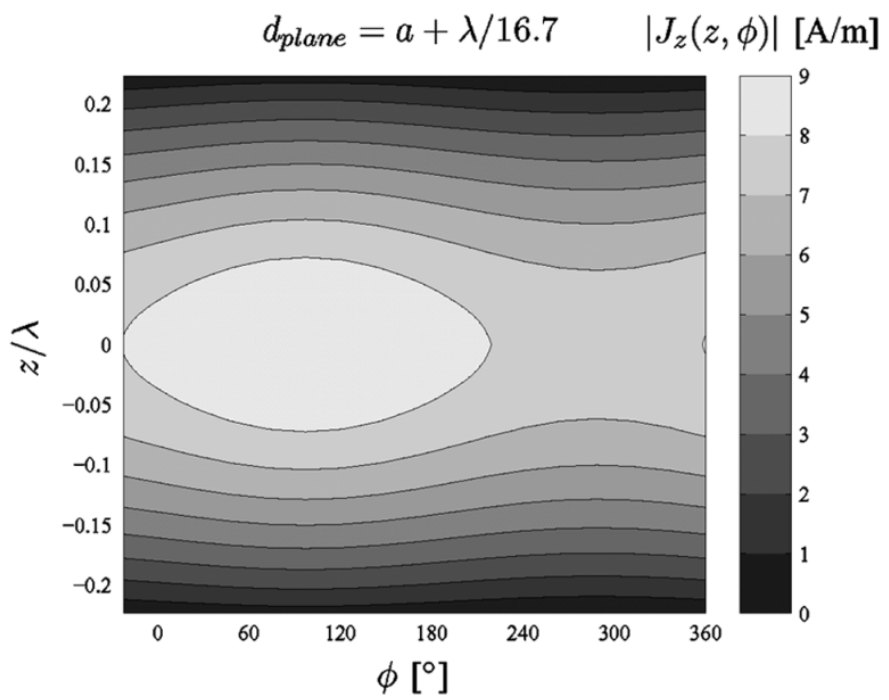

Fig. 9. Distribution of $\left|J_{z}(z, \phi)\right|$ over the cylindrical surface for $d_{\text {plane }}=$ $a+\lambda / 16.7$.

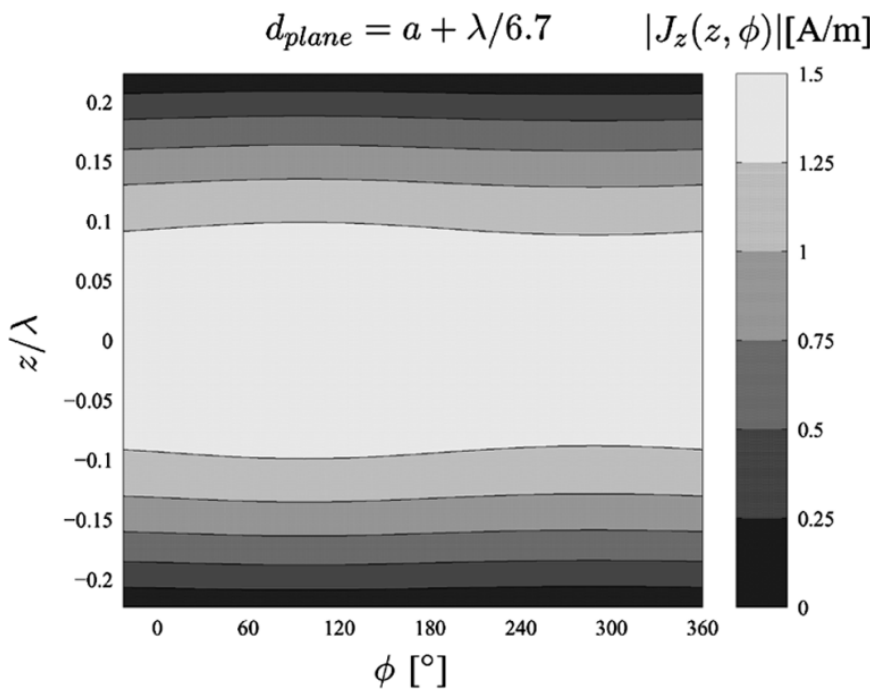

Fig. 10. Distribution of $\left|J_{z}(z, \phi)\right|$ over the cylindrical surface for $d_{\text {plane }}=$ $a+\lambda / 6.7$.

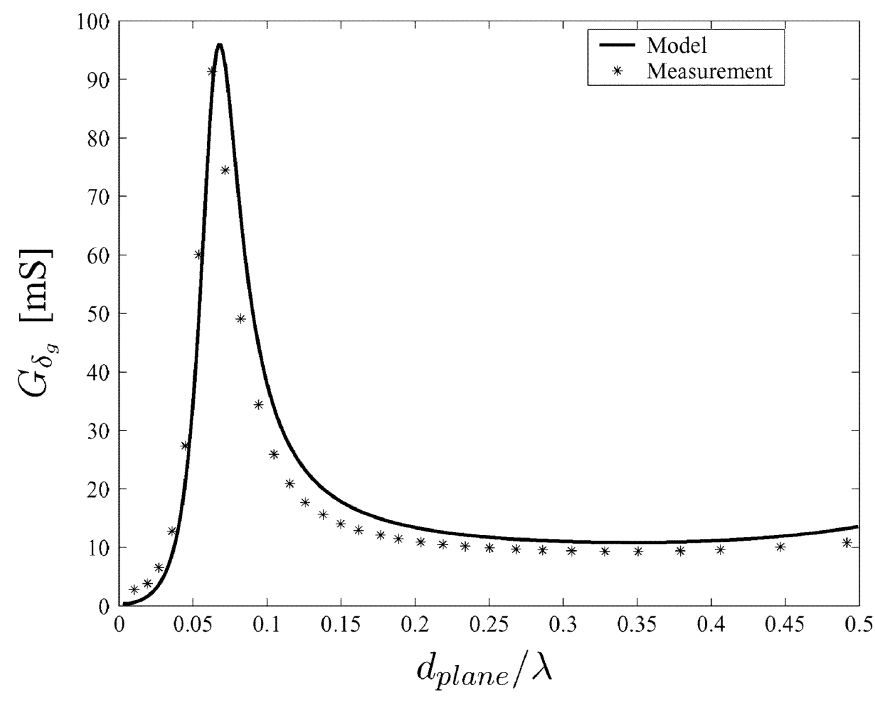

Fig. 11. Variation of the input conductance $G_{\delta_{g}}$ as a function of the distance to the plane.

physical point of view, this is not exactly correct, but we can investigate the influence of the circumferential surface currents 


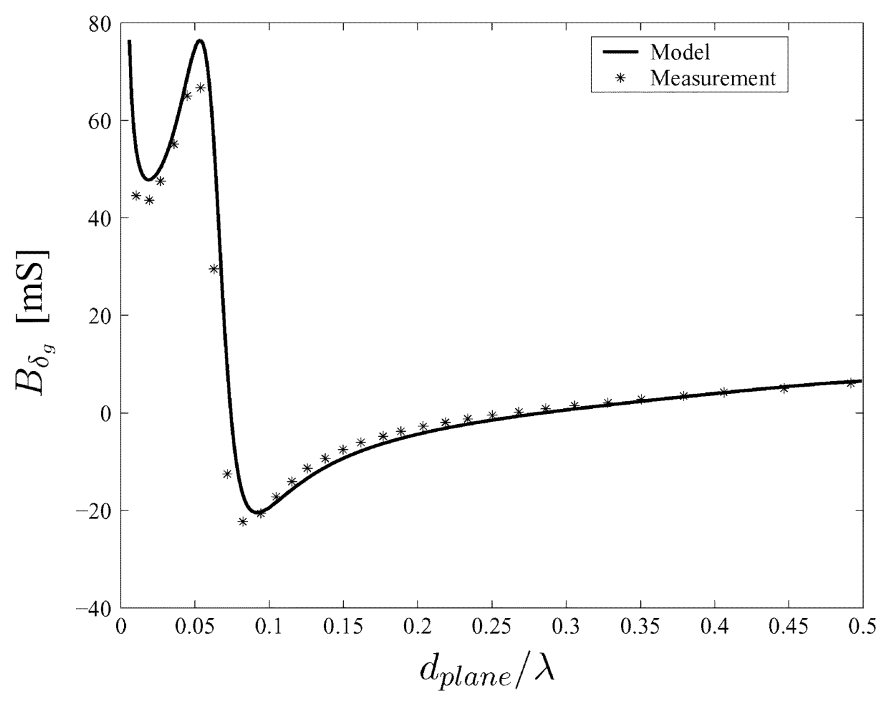

Fig. 12. Variation of the input susceptance $B_{\delta_{g}}$ as a function of the distance to the plane.

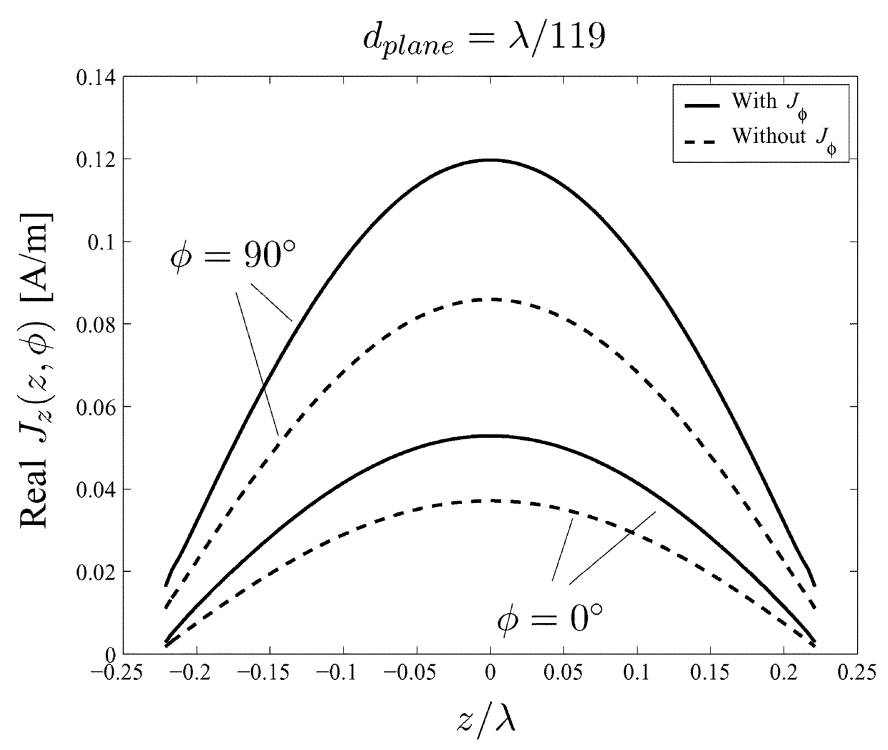

Fig. 13. Comparison of the real part of the longitudinal surface current at $\phi=$ $0^{\circ}$ and $\phi=90^{\circ}$ for $d_{\text {plane }}=\lambda / 119$ when $J_{\phi} \neq 0$ and $J_{\phi}=0$.

on $J_{z}(z, \phi)$. As a first example, we place the dipole very close to the plane, namely $d_{\text {plane }}=\lambda / 119$. At $900 \mathrm{MHz}$, this means that the cylindrical surface is as close as $1 \mathrm{~mm}$ from the plane. Figs. 13 and 14 show respectively the real and imaginary distribution of $J_{z}(z, \phi)$ for $\phi=0^{\circ}$ and $\phi=90^{\circ}$ when $J_{\phi}(z, \phi) \neq 0$ and $J_{\phi}(z, \phi)=0$. The difference is especially important for the real part of the longitudinal surface current. When one compares the magnitudes of the real and imaginary parts, it is clear that the latter is dominant. For this distance, $J_{\phi}(z, \phi)$ is only relevant near the end caps, but there is clearly a strong coupling between $J_{\phi}(z, \phi)$ and $J_{z}(z, \phi)$. Figs. 13 and 14 show the importance of this coupling. For larger distances, the interaction with the plane decreases and thus also the importance of the $J_{\phi}$-component, which is basically excited by the introduction of the plane that causes a rotational asymmetry in the configuration. We have found that for distances $d_{\text {plane }}$ beyond $\lambda / 50$, the calculation of $J_{\phi}(z, \phi)$ will again be unnecessary.

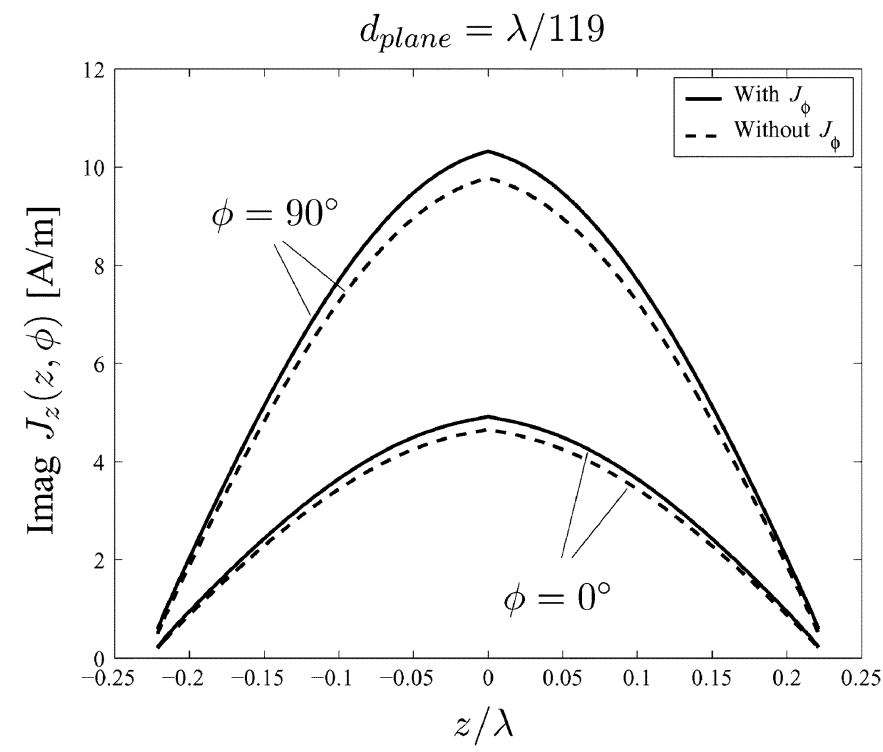

Fig. 14. Comparison of the imaginary part of the longitudinal surface current at $\phi=0^{\circ}$ and $\phi=90^{\circ}$ for $d_{\text {plane }}=\lambda / 119$ when $J_{\phi} \neq 0$ and $J_{\phi}=0$.

\section{CONCLUSION}

In this paper, an accurate model of a dipole antenna is constructed. The only approximation (or rather assumption) we made is that we impose a $z$-directed electrical field over the boundary of the gap region. It has been demonstrated mathematically that no circumferential current occurs if the antenna is in free-space. The kernel of the integral equation is exact, the physical boundary of the dipole is described exactly and the boundary conditions are imposed at the real surface of the dipole, in contrast with the classical methods to solve the dipole in free-space configuration.

As a worst case study of a dipole near a conducting object, we considered the configuration of a dipole close to an infinite PEC screen. A rigorous model was constructed to take the effect of the screen into account in the integral equation. A strong modification of the current distribution was observed. The results were compared with experimental data and excellent correspondence was obtained. For larger distances, the circumferential current component can be neglected.

\section{REFERENCES}

[1] R. W. P. King, "The linear antenna-Eighty years of progress," Proc. IEEE, vol. 55, pp. 2-16, Jan. 1967.

[2] J. D. Kraus, Antennas. New York: McGraw-Hill, 1950.

[3] R. F. Harrington, Field Computation by Moment Methods. Piscataway, NJ: IEEE Press, 1993.

[4] L. V. G. Miano and V. G. Vaccaro, "A hybrid procedure to solve Hallén's problem," IEEE Trans. Electromagn. Compat., vol. 38, pp. 495-498, Aug. 1996.

[5] G. P. Junker, A. A. Kishk, and A. W. Glisson, "A novel delta gap source model for center fed cylindrical dipoles," IEEE Trans. Antennas Propag., vol. 43, no. 5, pp. 537-540, May 1995.

[6] W. A. Imbriale and P. G. Ingerson, "On numerical convergence of moment solutions of moderately thick wire antennas using sinusoidal basis functions," IEEE Trans. Antennas Propag., vol. AP-21, no. 3, pp. 363-366, May 1973.

[7] R. E. Collin, "Equivalent line current for cylindrical dipole antennas and its asymptotic behavior," IEEE Trans. Antennas Propag., vol. AP-32, no. 2, pp. 200-204, Feb. 1984. 
[8] M. O. M. Douglas and M. A. Stuchly, "Accurate modeling of thin-wire antennas in the FDTD method," Microwave Opt. Tech. Lett., vol. 21, pp. 261-265, May 1999.

[9] S. Watanabe and M. Taki, "An improved FDTD model for the feeding gap of a thin-wire antenna," IEEE Microwave Guided Wave Lett., vol. 8, no. 4, pp. 152-154, Apr. 1998.

[10] H. J. Eom, J. K. Park, and Y. H. Cho, "A circular cylindrical dipole antenna," Microwave Opt. Tech. Lett., vol. 31, no. 4, pp. 322-324, Nov. 2001.

[11] F. M. Tesche, "The effect of the thin-wire approximation and the source gap model on the high-frequency integral equation solution of radiating antennas," IEEE Trans. Antennas Propag., vol. AP-20, no. 2, pp. 210-211, Mar. 1972.

[12] D. C. Chang, "On the electrically thick cylindrical antenna," Radio Sci., vol. 2, pp. 1043-1060, Sept. 1967.

[13] N. I. D. Wu and N. Kikuma, "Hallén type integral equation for cylindrical antennas with finite gap excitation," IEICE Trans. Commun., vol. E82-B, pp. 2145-2152, Dec. 1999.

[14] L. C. Shen, T. T. Wu, and R. W. P. King, "A simple formula of current in dipole antennas," IEEE Trans. Antennas Propag., vol. AP-16, no. 5, pp. 542-547, Sep. 1968.

[15] R. F. Harrington, "Straight wires with arbitrary excitation and loading," IEEE Trans. Antennas Propag., vol. 15, no. 4, pp. 502-515, 1967.

[16] R. H. MacPhie and S. K. Darbha, "The input impedance of a thin dipole with sinusoidal surface current distribution by the poynting vector method," IEEE Trans. Antennas Propag., vol. 43, no. 11, pp. 1336-1339, Nov. 1995.

[17] J. S. J. R. M. Makinen and M. A. Kivikoski, "An improved thin-wire model for FDTD," IEEE Trans. Antennas Propag., vol. 50, no. 5, pp. 1245-1255, May 2002.

[18] A. Rudge, "Input impedance of a dipole antenna above a conducting half space," IEEE Trans. Antennas Propag., vol. AP-20, no. 1, pp. 86-89, Jan. 1972.

[19] P. L. Christiansen, T. Itoh, E. K. Miller, A. J. Poggio, C. P. Wu, and R. Mittra, Computer Techniques for Electromagnetics and Antennas. Copenhagen, Denmark: Danish Association of Professional Engineers, 1971, pt. 1.

[20] T. J. Cui and W. C. Chew, "Accurate model of arbitrary wire antennas in free space, above, or inside ground," IEEE Trans. Antennas Propag., vol. 48, no. 4, pp. 482-493, Apr. 2000.

[21] J. A. Huffman and D. H. Werner, "Modeling of a cylindrical wire antenna with flat end caps using a rigorous moment method technique," Radio Sci., vol. 36, pp. 407-423, May-Jun. 2001.

[22] E. Jørgensen, "Higher-order integral equation methods in computational electromagnetics," Ph.D. dissertation, Technical University of Denmark, Ørsted, Denmark, May 2003.

[23] D. Jones, Methods in Electromagnetic Wave Propagation. Oxford, U.K.: Clarendon Press, 1994.

[24] A. Peterson, S. Ray, and R. Mittra, Computational Methods for Electromagnetics. Piscataway, NJ: IEEE, 1998.

[25] A. Poggio and E. Miller, Integral Equation Solutions of Three-Dimensional Scattering Problems. Oxford, U.K.: Pergamon, 1973.
[26] R. F. Harrington, Time-Harmonic Electromagnetic Fields. New York: McGraw-Hill, 1961.

[27] A. W. Glisson, "Equivalent current excitation for an aperture antenna embedded in an arbitrarly shaped impedance surface," IEEE Trans. Antennas Propag., vol. 50, no. 7, pp. 966-970, Jul. 2002.

[28] G. Burke and A. Poggio, Numerical Electromagnetics Code (NEC)Method of Moments, Part 2: Program Description-Code, 1981. [Online]. Available.

[29] N. Stevens and L. Martens, "An efficient method to calculate surface currents on a PEC cylinder with flat end caps," Radio Sci., vol. 38, pp. 6-1-6-10, Jan. 2003.

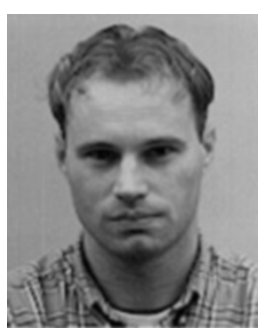

Nobby Stevens received the master's degree in physical engineering and the Ph.D. degree from Ghent University, Ghent, Belgium, in 1997 and May 2004, respectively. He finished a DEA formation at the Institut National Polytechnique de Grenoble, Grenoble, France, in 1997.

From the end of 1997 until August 1998, he worked as a Product Development Engineer at Philips. In August 1998, he was performing research on numerical modeling of electromagnetic fields interacting with the human body at the Department of Information Technology of Ghent University. In June 2004, he joined Agilent EEsof EDA, Ghent, Belgium, as a Research and Development Engineer.

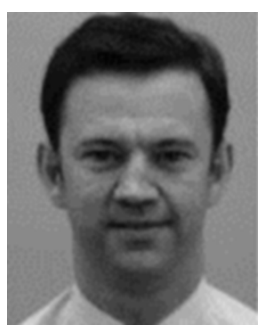

Luc Martens (M'91) was born in Ghent, Belgium, on May 14, 1963. He received the M.Sc. degree in electrical engineering and the Ph.D. degree from Ghent University, Ghent, in July 1986 and December 1990, respectively.

From September 1986 to December 1990, he was a Research Assistant at the Department of Information Technology (INTEC) at Ghent University. During this period, his scientific work was focused on the physical aspects of hyperthermic cancer therapy. His research work dealt with electromagnetic and thermal modeling and with the development of measurement systems for that application. Since January 1991, he has been a Member of permanent staff of the Interuniversity MicroElectronics Centre (IMEC), Leuven, Belgium and is responsible for the research on characterization of packaging technologies with respect to high-frequency and EMC behavior and for the research on experimental modeling of active devices at INTEC. He is also further studying topics related to the health effects of wireless communication devices. Since April 1993, he has been a Professor of electrical applications of electromagnetism at Ghent University. 